\title{
Antiviral therapy reduces rebleeding rate in patients with hepatitis B-related cirrhosis with acute variceal bleeding after endotherapy
}

Lingling $\mathrm{He}^{1+}{ }^{1+}$, Xiaohui $\mathrm{Ye}^{2+}$, Jiali Ma ${ }^{1}$, Ping $\mathrm{Li}^{1}$, Yu Jiang ${ }^{1}$, Julong $\mathrm{Hu}^{1}$, Junru Yang ${ }^{1}$, Yuling Zhou ${ }^{1}$, Xiuxia Liang ${ }^{1}$, Yijun Lin ${ }^{1}$ and Hongshan Wei ${ }^{i^{*}}$ (i)

\begin{abstract}
Background: The preventive effects of antiviral therapy to reduce rebleeding rate in patients with hepatitis Brelated cirrhosis undergoing endoscopic treatment have not yet been reported.

Methods: In this retrospective cohort study, 1139 patients with chronic hepatitis B with first acute variceal bleeding after endoscopic therapy from September 2008 to December 2017 were included. Among them, 923 who received and 216 who did not receive antiviral therapy were followed up for rebleeding. Cumulative rebleeding rate was calculated using the Kaplan-Meier method. Univariate and multivariate logistic regression analyses were performed to estimate the effects of antiviral therapy on rebleeding risk. The propensity score matched method and inverse probability of treatment weighting analysis were used to calculate the rebleeding rate between the antiviral and non-antiviral groups.
\end{abstract}

Results: The rebleeding rates were $40.5,60.7,72.6$, and $89.2 \%$ in antiviral group at 1, 2, 3, and 5 years, respectively. The corresponding rebleeding rates in the non-antiviral group were 54.2, 72.4, 84.4, and 93.3\%, respectively. The multivariate logistic regression analysis revealed that antiviral therapy was an independent protective factor associated with rebleeding.

Conclusion: Antiviral treatment significantly reduced rebleeding rate in patients with HBV-related cirrhosis who received endoscopic treatment after the first variceal bleeding.

Keywords: Hepatitis virus B, Antiviral treatment, Gastroesophageal varices, Endoscopic treatment, Liver cirrhosis, Rebleeding rate

\section{Background}

Chronic hepatitis B virus (HBV) infection is a critical cause of liver cirrhosis and hepatocellular carcinoma worldwide $[1,2]$. As one of the regions with the highest rate of HBV infection in the world, nearly $40 \%$ of subjects with chronic $\mathrm{HBV}$ are living in China currently, although the vaccination strategies have decreased the number of chronic HBV patients [3]. About 350 million people worldwide suffer from

\footnotetext{
* Correspondence: drwei@ccmu.edu.cn

${ }^{\dagger}$ Lingling He and Xiaohui Ye contributed equally to this work.

'Department of gastroenterology, Beijing Ditan Hospital, Capital Medical University, No.8, Jingshun East Street, Chaoyang District, Beijing 100015, China

Full list of author information is available at the end of the article
}

chronic HBV infection, and HBV infection in China is the main cause of $\mathrm{HCC}[4,5]$. Although long-term antiviral therapy may prevent or delay the development of cirrhosis and its complications, it remains a global major public health burden, especially in China and other developing countries $[6,7]$. Without antiviral therapy in patients with HBV-related cirrhosis, the 5-year cumulative incidence of cirrhosis ranges from 8 to $20 \%$, and 5-year survival rate in those with untreated decompensated cirrhosis was low as $15 \%$, according to the present clinical guidelines $[8,9]$.

Acute gastroesophageal variceal bleeding is a major complication of cirrhosis that leads to a high mortality rate $(40 \%)$ and rebleeding rate ( $60 \%$ of survivors) $[10$, 
11]. Therefore, prevention of bleeding in cirrhotic patients with gastroesophageal varices is one of the major therapeutic goals [12]. Most guidelines recommended that drug and endoscopic therapies should be combined for the initial treatment of acute variceal bleeding [13]. Based on a recent report, the mortality of acute esophageal variceal bleeding increases nearly up to $20 \%$ in the recent years even after using the first-line therapy, such as endoscopic varices ligation (EVL) or endoscopic varices sclerotherapy (EVS) [14]. To prevent rebleeding, several drugs were used to improve portal hypertension, in which non-selective beta-blockers (NSBBs) remained the cornerstone, albeit carvedilol seemed more effective in decreasing portal pressure $[15,16]$. However, these drugs were all scrutinized in patients with severe or advanced cirrhosis [16].

Antiviral therapy was widely used to control the progression of chronic hepatitis $\mathrm{B}(\mathrm{CHB})$ during the past two decades. However, most of the current literatures focus on the immune active phases of chronic HBV infection [17, 18]; therefore, the effects of antiviral therapy on portal hypertension and gastroesophageal bleeding remain to be elucidated. In the present retrospective cohort study, we analyzed 1139 patients with $\mathrm{CHB}$ with acute variceal bleeding and found that antiviral therapy significantly deceased the variceal rebleeding rate, with higher survival rate.

\section{Methods}

\section{Patients and design}

This retrospective cohort study was conducted from 2008 to 2017 on all CHB patients with liver cirrhosis and acute variceal bleeding after an endoscopic therapy who were followed up in Capital Medical University affiliated Beijing Ditan Hospital. The exclusion criteria were as follows, i) patients coinfected with hepatitis $\mathrm{C}$ virus, alcoholic liver disease, and other chronic liver diseases; ii) patients with serious concurrent illness; iii) patients recurrent acute variceal bleeding or who received preventive endoscopic treatment.

These patients were chronically monoinfected with HBV who had HBV surface antigen (HBsAg)-positive for at least 6 months [19]. Liver cirrhosis was defined as the appearance of an irregular and nodular liver by two images, with impaired liver synthetic function. The normal structure of the liver lobule is severely damaged, with the evidence of the small and shrunken liver, splenomegaly and portal hypertension [20]. Acute variceal bleeding was defined as hematemesis or melena with blood pressure decreased by $20 \mathrm{mmHg}$. The study was approved by the Clinical Research Ethics Committee of Beijing Ditan Hospital.

\section{Clinical data collection and follow-up}

Clinical data, such as age, gender, diabetes, alcohol consumption, ascites, white blood cell (WBC), hemoglobin (HGB), platelet (PLT), alanine aminotransferase (ALT), aspartate aminotransferase(AST), total bilirubin (TBIL), albumin (ALB), gamma-glutamyl transpeptidase (GGT), alkaline phosphatase (ALP), cholinesterase (CHE), creatinine $(\mathrm{Cr})$, alpha fetoprotein (AFP), prothrombin time (PT), portal vein diameter, and spleen thickness, were collected at the time of acute variceal bleeding. The Child-Turcotte-Pugh score and model for end-stage liver disease (MELD) score were also recorded. The data was collected by two physicians alone, and checked by the third person.

All included patients were followed up for rebleeding and survival. The primary outcome was rebleeding rate at 1 year. Other outcomes were rebleeding rate at $2,3,4$ and 5 years and cumulative survival rate at 1 , $2,3,4$ and 5 years.

\section{Antiviral and endoscopic therapy}

Strategies for antiviral treatment, including administration of lamivudine, adefovir, telbivudine, entecavir, and tenofovir alone or combined, were based on the APASL guidelines and its update for the management of HBV infection [19]. Patients who received antiviral therapy before or after endoscopic treatment were all recorded.

For the endoscopic treatment of variceal bleeding, the standard EVL or EVS was performed based on the previous reports [21, 22]. Endoscopic therapies were based on Chinese guidelines: i) endoscopic variceal ligation was performed for patients with esophageal variceal bleeding; ii) cyanoacrylate was injected for the patients with gastric variceal hemorrhage; and iii) the "sandwich therapy" was used for patients with esophagealgastric variceal hemorrhage. The strategy of "sandwich therapy" was as follows: $2 \mathrm{ml}$ polidocanol $+0.5 \mathrm{ml}$-butyl- 2 cyanoacrylate $+2 \mathrm{ml}$ polidocanol. The injection might be repeated as necessary as previously report [23].

\section{Statistical analysis}

The statistical analysis was performed using the SPSS version 19.0 (IBM Corp., Armonk, NY, United States) and $\mathrm{R}$ program (version 3.5.1, Vietna, Austria) [24]. Quantitative data were summarized with mean \pm standard deviation (SD) or median with interquartile range, and their distributions in the two groups were compared using two-sample $t$ test or Mann-Whitney U test. Qualitative data were summarized with frequency (percent) and analyzed using the chi-square test. Cumulative rebleeding and survival rates were calculated and plotted using the Kaplan-Meier method. Log-rank test was used to examine differences in rebleeding and survival rates between the antiviral and non-antiviral groups. Logistic 
regression analysis was performed, and odds ratio (OR) was calculated to identify variables associated with rebleeding risk. Multivariate analysis was performed with variables that showed association in the univariate analysis. Both unadjusted and adjusted ORs and 95\% confidence intervals were obtained. A two-sided $P<0.05$ was considered statistically significant.

Propensity score matching method (PSM) is associated with reduced systematic selection bias, so this method is considered as a kind of randomization for retrospective study. We used the propensity score matching method to reduce significant differences in demographics between the antiviral and non-antiviral groups. Using the multiple logistic regression analysis, a propensity score was estimated for all patients. Variables used in the model included age, sex, diabetes, alcohol consumption, ascites, WBC, PLT, HGB, ALT, AST, TBIL, GGT, ALB, Cr, PT, AFP, hepatitis B eantigen (HBeAg), and MELD score. We performed caliper matching on the PS (nearest available matching). Pairs (antiviral and non-antiviral groups) on the PS logit were matched within a range of 0.2 SD. Rebleeding risk was calculated using the propensity score matched cohort. Inverse probability of treatment weighting (IPTW) analysis was performed to calculate rebleeding and survival rates to adjust data bias.

\section{Results \\ Baseline characteristics}

A total of 3179 patients with HBV-related cirrhosis who underwent endoscopic therapy from September 2008 to December 2017 were reviewed. After excluding patients with recurrent acute variceal bleeding $(n=1917)$, who received endoscopic treatment to prevent bleeding $(n=31)$, who were lost to follow-up $(n=92)$, the final analysis consecutively included 1139 patients with $\mathrm{CHB}$ with first occurrence of acute variceal bleeding and received endoscopic hemostasis. Among them, 923 patients received antiviral therapy and 216 did not (Fig. 1). The latter group consisted of patients who refused to receive antiviral medications. The patients were followed up for 1-9years, with an average of 3.2 years.

Of the 1139 patients who were followed up over 1 year, rebleeding occurred in 491 (43.1\%) after the endoscopic therapy, and 374 (40.5\%) of the 923 patients who received antiviral therapy had rebleeding, whereas 117 (54.2\%) of the 216 patients who did not receive antiviral therapy had rebleeding. The rebleeding rate was significantly higher in

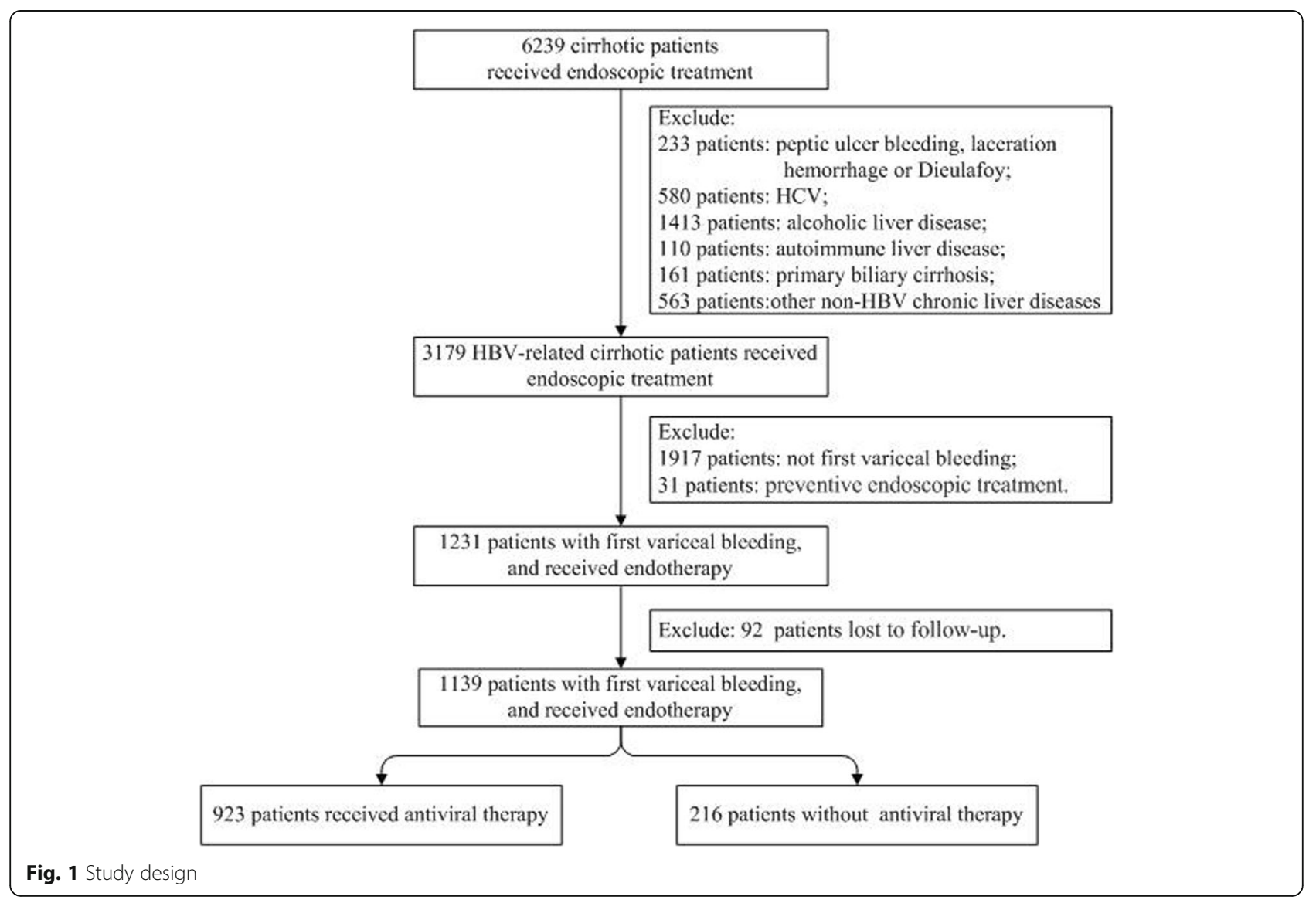


patients who did not receive antiviral therapy $(p<0.001)$. The baseline characteristics are shown in Table 1 . The antiviral drugs are listed in Table 2.

The 1139 patients consisted of 847 men and 292 women, with the mean age of $51.2 \pm 10.3$ months. Patients who had rebleeding consisted of a smaller proportion from the antiviral group, had higher diabetes rate, higher ascites rate, lower HGB, higher GGT, and a large proportion of CTP class C.

In the analysis, HBV-DNA was detected in 718 patients. In patients who were HBV-DNA positive, the rebleeding rates at 1-, 3-, and 5-year follow-up were $40.2,70.6$, and $91.3 \%$, respectively. In patients who were HBV-DNA negative, the rebleeding rates at 1-, 3-, and 5-year follow-up were 41.5, 76.3, and 92.4\%, respectively. The difference in rebleeding rate between patients with positive and negative HBV-DNA was not significant. At the same time, the survival rates of patients with positive HBV-DNA at 1-, 3-, and 5-year follow-up were 94.4, 88.0 , and $47.3 \%$, respectively, whereas in those with negative HBV-DNA, they were 97.0, 87.9, and 52.3\%,
Table 2 Antiviral drugs used in patients

\begin{tabular}{ll}
\hline Drug & $\mathrm{N}(\%)$ \\
\hline Adefovir dipivoxil & $188(20.4)$ \\
Lamivudine & $86(9.3)$ \\
Entecavir & $490(53.1)$ \\
Telbivudine & $9(1.0)$ \\
Tenofovir & $12(1.3)$ \\
Adefovir + Lamivudine & $77(8.3)$ \\
Adefovir + Entecavir & $43(4.7)$ \\
Adefovir + Telbivudine & $17(1.8)$ \\
Entecavir + Tenofovir & $1(0.1)$ \\
\hline
\end{tabular}

respectively. The difference in survival rate between patients with positive and negative HBV-DNA was also not significant.

The mean age of all patients was $51.2 \pm 10.3$ months. The difference in rebleeding and survival rates at 1 year based on patients' age was not significant. However, significant differences were observed in the rebleeding and

Table 1 Baseline characteristics

\begin{tabular}{|c|c|c|c|c|c|c|}
\hline \multirow[t]{2}{*}{ Characteristics } & \multicolumn{3}{|l|}{ Before matching $(N=1139)$} & \multicolumn{3}{|l|}{ After matching $(\mathrm{N}=348)$} \\
\hline & No rebleeding $(N=648)$ & Rebleeding $(N=491)$ & SD & No rebleeding $(N=192)$ & Rebleeding $(N=156)$ & SD \\
\hline Age $(y r)$ & $50.7 \pm 10.3$ & $51.8 \pm 10.4$ & -1.168 & $51.8 \pm 10.6$ & $51.8 \pm 11.5$ & -0.019 \\
\hline Male sex & $483(74.5 \%)$ & $364(74.1 \%)$ & 0.024 & $123(64.1 \%)$ & $98(62.8 \%)$ & 0.057 \\
\hline Antiviral therapy & $549(84.7 \%)$ & $374(76.2 \%)$ & 13.291 & 109 (56.8\%) & $65(41.7 \%)$ & 7.854 \\
\hline Diabetes & $117(18.1 \%)$ & $140(28.5 \%)$ & 17.484 & $19(9.9 \%)$ & $35(22.4 \%)$ & 10.324 \\
\hline Alcohol consumption & $18(2.8 \%)$ & $16(3.3 \%)$ & 0.223 & $2(1.0 \%)$ & $3(1.9 \%)$ & 0.472 \\
\hline Ascites & $479(73.9 \%)$ & $419(85.3 \%)$ & 21.824 & $142(74.0 \%)$ & $136(87.2 \%)$ & 9.363 \\
\hline WBC $\left(\times 10^{9} / L\right)$ & $4.0(2.7,6.4)$ & $4.3(3.0,6.2)$ & -0.036 & $4.5(3.0,7.5)$ & $4.8(3.3,7.0)$ & 0.268 \\
\hline $\operatorname{PLT}\left(\times 10^{9} / \mathrm{L}\right)$ & $62.0(44.4,89.0)$ & $66.9(46.4,94.0)$ & -5.554 & $66.0(44.4,97.8)$ & $73.5(52.5,111.8)$ & -6.968 \\
\hline $\mathrm{HGB}(\mathrm{g} / \mathrm{l})$ & $89.2 \pm 25.2$ & $84.1 \pm 25.4$ & 5.075 & $81.2(67.4,101.0)$ & $77.7(60.2,93.2)$ & 5.946 \\
\hline $\mathrm{ALT}(\mathrm{U} / \mathrm{L})$ & $23.1(17.1,32.8)$ & $22.5(17.0,33.0)$ & -3.724 & $22.2(17.0,32.6)$ & $21.7(16.2,33.0)$ & -4.208 \\
\hline AST (U/L) & $28.6(21.7,39.7)$ & $29.0(22.4,41.0)$ & -10.332 & $27.5(21.6,39.8)$ & $28.9(22.4,39.5)$ & -13.064 \\
\hline TBIL $(\mu \mathrm{mol} / \mathrm{l})$ & $17.9(12.8,25.3)$ & $18.1(11.9,25.1)$ & -2.604 & $16.7(11.9,23.8)$ & $17.1(10.9,24.4)$ & -5.402 \\
\hline GGT (U/L) & $19.2(12.9,31.2)$ & $26.4(15.6,45.3)$ & -14.324 & $20.1(12.4,33.5)$ & $26.9(14.1,43.5)$ & -15.883 \\
\hline ALB $(g / l)$ & $33.1 \pm 5.8$ & $32.7 \pm 5.7$ & 0.383 & $31.9 \pm 5.4$ & $31.8 \pm 5.7$ & 0.163 \\
\hline $\mathrm{Cr}(\mu \mathrm{mol} / \mathrm{l})$ & $63.3(53.7,74.3)$ & $63.9(53.4,75.8)$ & -0.539 & $58.9(48.6,69.4)$ & $59.4(48.9,72.3)$ & -1.935 \\
\hline PT (s) & $14.9(13.9,16.2)$ & $15.0(13.7,16.4)$ & -0.076 & $15.0(14.0,16.5)$ & $15.3(14.2,16.8)$ & -0.516 \\
\hline AFP (ng/ml) & $2.8(1.8,5.3)$ & $3.1(1.7,5.9)$ & -1.990 & $2.8(1.8,5.3)$ & $3.3(1.7,5.9)$ & -0.955 \\
\hline HBeAg (positive) & 146 (26.9\%) & $103(25.6 \%)$ & 0.211 & $21(10.9 \%)$ & $21(13.5 \%)$ & -0.025 \\
\hline HBV-DNA (positive) & $128(30.3 \%)$ & $86(29.2 \%)$ & 0.102 & $34(26.0 \%)$ & $26(28.0 \%)$ & 0.111 \\
\hline MELD score & $6.1 \pm 4.2$ & $6.3 \pm 4.9$ & -0.194 & $4.8 \pm 4.8$ & $5.7 \pm 5.4$ & -0.881 \\
\hline CTP class (A/B/C) & $177 / 359 / 112$ & $81 / 296 / 114$ & 20.548 & $44 / 113 / 35$ & 20/95/41 & 7.386 \\
\hline
\end{tabular}

Data are presented as mean \pm standard deviation, or number (percentage). In the analysis, 946 patients detected HBeAg.718 patients detected HBV-DNA, 224 patients detected HBV-DNA after matching

SD standardised difference, WBC white blood cell, $P L T$ Platelet, HGB hemoglobin, ALT alanine aminotransferase, TBIL total bilirubin, GGT gamma-glutamyl transpeptidase, $A L B$ albumin, $C r$ creatinine, $P T$ prothrombin time, $A F P$ alpha fetoprotein, $H B e A g$ hepatitis $B$ virus e antigen, $H B V$-DNA hepatitis b virus deoxyribonucleic acid, CTP class Child-Turcotte-Pugh class, MELD model for end-stage liver disease 
survival rates at 3 and 5 years, i.e., elderly patients were more likely to rebleed and die.

Before the propensity score matching, differences in sex, WBC, PLT, HGB, ALB, Cr, PT, AFP, HBeAg, and MELD score were significant in the entire cohort. After matching, the variables above were balanced in the propensity score matched cohort (348 patients) (Table 3).

\section{Factors associated with rebleeding}

To estimate the effects of antiviral therapy on rebleeding risk, we performed univariate and multivariate logistic regression analyses. The univariate logistic regression analysis revealed that the antiviral therapy, diabetes, ascites, LY\%, HGB, AST, GGT, CHE, AFP, and CTP class were factors associated with rebleeding. Among these factors, antiviral therapy, diabetes, ascites, HGB, and GGT were independent factors according to the multivariate logistic regression analysis (Table 4).
Benefits of antiviral treatment on rebleeding and survival The cumulative incidence rates of rebleeding in different time points were analyzed. The rebleeding rate in the antiviral group was lower than that in the non-antiviral group at 1, 2, 3, 4 and 5 years $(P<0.01)$ (Fig. 2a, b, c, d). The rebleeding rates were 40.5, 60.7, 72.6, 82.3 and $89.2 \%$ in the antiviral group at $1,2,3,4$ and 5 years, respectively. The corresponding rebleeding rates in the non-antiviral group were 54.2, 72.4, 84.4, 89.7 and $93.3 \%$, respectively. The antiviral treatment significantly reduced the rebleeding rate.

In the propensity score matched cohort, the cumulative incidence rates of rebleeding were also lower in the antiviral group (174 patients) than that in the nonantiviral group (174 patients) at $1(P=0.002), 2(P=$ $0.019), 3(P=0.010)$, and 5 years $(P=0.028)$ (Fig. $2 \mathrm{e}, \mathrm{f}, \mathrm{g}$, h).

To further confirm the benefits of antiviral treatment, patients who received them $>1$ year $(n=529)$ and $<1$ year $(n=394)$ were analyzed. The cumulative incidence

Table 3 Baseline characteristics of the entire cohort and propensity score matched cohort

\begin{tabular}{|c|c|c|c|c|c|c|}
\hline \multirow[t]{2}{*}{ Characteristics } & \multicolumn{3}{|l|}{ Before matching $(\mathrm{N}=1139)$} & \multicolumn{3}{|l|}{ After matching $(\mathrm{N}=348)$} \\
\hline & $\begin{array}{l}\text { Non-antiviral group ( } \mathrm{N}= \\
216)\end{array}$ & $\begin{array}{l}\text { Antiviral group }(N= \\
\text { 923) }\end{array}$ & SD & $\begin{array}{l}\text { Non-antiviral group }(N= \\
174)\end{array}$ & $\begin{array}{l}\text { Antiviral group }(\mathrm{N}= \\
\text { 174) }\end{array}$ & SD \\
\hline$\overline{\text { Age }(y r)}$ & $51.5 \pm 11.5$ & $51.1 \pm 10.1$ & 0.336 & $52.1 \pm 11.2$ & $51.5 \pm 10.8$ & 0.626 \\
\hline Male sex & $144(66.7 \%)$ & $703(76.2 \%)$ & 8.283 & $113(64.9 \%)$ & $108(62.1 \%)$ & 0.310 \\
\hline Diabetes & $40(18.5 \%)$ & $217(23.5 \%)$ & 2.496 & $29(16.7 \%)$ & $25(14.4 \%)$ & 0.351 \\
\hline $\begin{array}{l}\text { Alcohol } \\
\text { consumption }\end{array}$ & $6(2.8 \%)$ & $28(3.0 \%)$ & 0.040 & $3(1.7 \%)$ & $2(1.1 \%)$ & 0.203 \\
\hline Ascites & $176(81.5 \%)$ & $722(78.2 \%)$ & 1.114 & $138(79.3 \%)$ & $140(80.5 \%)$ & 0.072 \\
\hline WBC $(\times 109 / L)$ & $4.5(3.0,7.0)$ & $4.1(2.8,6.2)$ & 0.418 & $4.6(3.1,7.2)$ & $4.8(3.1,7.3)$ & -0.309 \\
\hline PLT (×109/L) & $69.0(44.4,119.0)$ & $63.0(45.4,88.4)$ & 15.353 & $68.5(44.4,119.2)$ & $69.0(49.4,96.3)$ & 5.467 \\
\hline HGB $(g / l)$ & $80.0 \pm 23.7$ & $88.7 \pm 25.5$ & -8.659 & $81.0 \pm 23.5$ & $82.2 \pm 22.9$ & -1.163 \\
\hline $\mathrm{ALT}(\mathrm{U} / \mathrm{L})$ & $22.5(16.8,32.6)$ & $22.9(17.1,33.0)$ & -3.452 & $22.8(16.6,32.9)$ & $21.4(16.5,32.8)$ & 0.464 \\
\hline AST (U/L) & $28.4(22.3,41.2)$ & $29.3(21.9,41.2)$ & -1.642 & $28.4(21.8,40.1)$ & $28.0(21.8,39.4)$ & -0.006 \\
\hline TBIL $(\mu \mathrm{mol} / \mathrm{l})$ & $18.1(11.9,24.9)$ & $18.1(12.6,25.2)$ & -2.486 & $17.3(11.4,24.6)$ & $16.1(11.2,23.7)$ & -1.240 \\
\hline GGT (U/L) & $22.6(12.7,44.0)$ & $20.7(13.8,35.6)$ & 3.292 & $23.4(13.1,37.3)$ & $21.6(13.3,33.5)$ & 1.455 \\
\hline ALB (g/l) & $31.5 \pm 5.3$ & $33.3 \pm 5.8$ & -1.800 & $31.9 \pm 5.2$ & $31.9 \pm 5.8$ & -0.039 \\
\hline $\mathrm{Cr}(\mu \mathrm{mol} / \mathrm{l})$ & $59.2(48.5,72.3)$ & $64.2(54.6,75.4)$ & -2.655 & $58.5(48.6,72.1)$ & $59.9(48.8,70.6)$ & 4.031 \\
\hline PT (s) & $15.3(14.1,16.7)$ & $14.9(13.7,16.2)$ & 0.332 & $15.2(14.1,16.5)$ & $15.3(14.0,16.6)$ & -0.100 \\
\hline AFP $(n g / m l)$ & $2.5(1.6,4.6)$ & $3.0(1.8,5.8)$ & -2.205 & $2.5(1.6,4.6)$ & $3.3(2.0,5.9)$ & -2.293 \\
\hline HBeAg (positive) & $18(10.3 \%)$ & 231 (30.0\%) & 28.471 & $18(10.3 \%)$ & $24(13.8 \%)$ & 0.975 \\
\hline HBV-DNA (positive) & $28(23.7 \%)$ & $186(31.0 \%)$ & 2.492 & $25(24 \%)$ & $35(29.2)$ & 0.747 \\
\hline MELD score & $5.6(2.7,8.4)$ & $6.2(3.9,8.8)$ & -0.910 & $5.4(2.6,8.3)$ & $5.0(1.8,8.2)$ & 0.430 \\
\hline CTP class (A/B/C) & $39 / 130 / 47$ & 219/525/179 & 3.312 & $35 / 106 / 33$ & 29/102/43 & 1.955 \\
\hline
\end{tabular}

Data are presented as mean \pm standard deviation, or number (percentage). In the entire cohort, 718 patients detected HBV-DNA, 224 patients detected HBV-DNA after matching

SD standardised difference, WBC white blood cell, PLT Platelet, HGB hemoglobin, ALT alanine aminotransferase, TBIL total bilirubin, GGT gamma-glutamyl transpeptidase, $A L B$ albumin, $C r$ creatinine, $P T$ prothrombin time, $A F P$ alpha fetoprotein, $H B e A g$ hepatitis $B$ virus e antigen, $H B V$-DNA hepatitis $b$ virus deoxyribonucleic acid, CTP class Child-Turcotte-Pugh class, MELD model for end-stage liver disease 
Table 4 Factors associated with rebleeding

\begin{tabular}{|c|c|c|c|c|}
\hline Variable & Univariate analysis OR (95\%Cl) & $p$ & Multivariate analysis OR $(95 \% \mathrm{Cl})$ & $p$ \\
\hline Age (yr) & $1.011(1.000-1.023)$ & 0.059 & & \\
\hline Gender & $1.021(0.781-1.336)$ & 0.878 & & \\
\hline Antiviral therapy & $0.576(0.428-0.777)$ & $<0.001$ & $0.563(0.389-0.817)$ & 0.002 \\
\hline Diabetes & $1.810(1.368-2.395)$ & $<0.001$ & $1.893(1.347-2.662)$ & $<0.001$ \\
\hline Alcohol consumption & $1.179(0.595-2.336)$ & 0.637 & & \\
\hline Ascites & $2.053(1.513-2.787)$ & $<0.001$ & $1.820(1.253-2.642)$ & 0.002 \\
\hline WBC $\left(\times 10^{9} / \mathrm{L}\right)$ & $1.003(0.969-1.039)$ & 0.858 & & \\
\hline LY\% & $0.986(0.974-0.998)$ & 0.026 & & \\
\hline $\operatorname{PLT}\left(\times 10^{9} / \mathrm{L}\right)$ & $1.002(1.000-1.004)$ & 0.122 & & \\
\hline HGB $(g / l)$ & $0.992(0.987-0.997)$ & 0.001 & $0.994(0.988-0.999)$ & 0.032 \\
\hline $\operatorname{ALT}(\mathrm{U} / \mathrm{L})$ & $1.001(0.999-1.004)$ & 0.241 & & \\
\hline AST (U/L) & $1.003(1.001-1.006)$ & 0.012 & & \\
\hline GGT (U/L) & $1.011(1.006-1.015)$ & $<0.001$ & $1.011(1.006-1.016)$ & $<0.001$ \\
\hline TBIL $(\mu \mathrm{mol} / \mathrm{l})$ & $1.004(0.999-1.008)$ & 0.124 & & \\
\hline CHE (U/L) & $1.000(1.000-1.000)$ & 0.014 & & \\
\hline HBeAg (positive) & $0.934(0.696-1.252)$ & 0.646 & & \\
\hline HBV-DNA (positive) & $0.948(0.685-1.313)$ & 0.750 & & \\
\hline $\operatorname{AFP}(n g / m l)$ & $1.003(1.001-1.005)$ & 0.007 & & \\
\hline MELD score & $1.010(0.984-1.036)$ & 0.474 & & \\
\hline CTP class & $1.492(1.242-1.792)$ & $<0.001$ & & \\
\hline
\end{tabular}

WBC white blood cell, LY\% percentage of lymphocytes, PLT Platelet, ALT alanine aminotransferase, GGT gamma-glutamyl transpeptidase, CHE cholinesterase, AFP alpha fetoprotein, HBeAg hepatitis B virus e antigen, HBV-DNA hepatitis b virus deoxyribonucleic acid, CTP class Child-Turcotte-Pugh class, MELD model for endstage liver disease

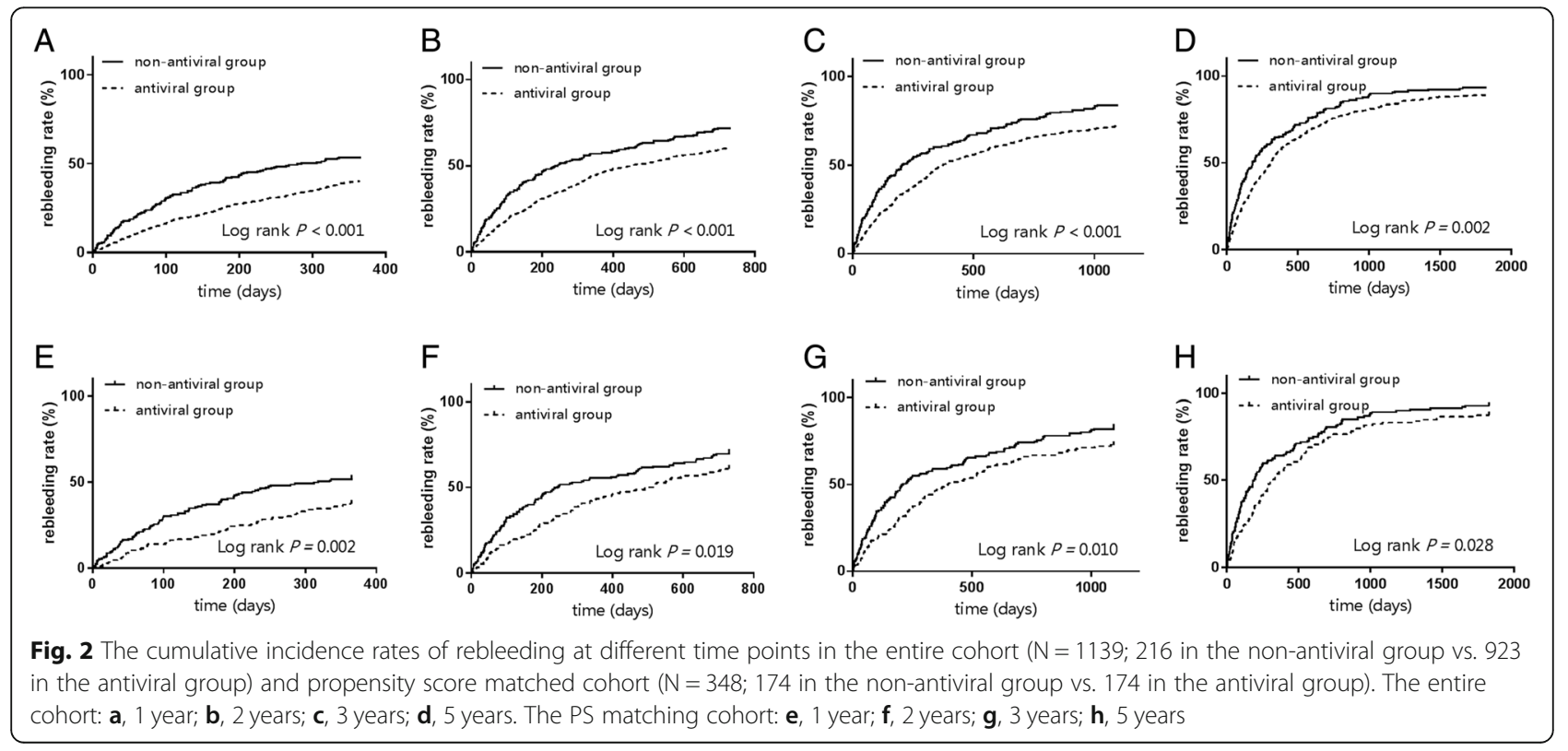


rate of rebleeding in patients who received antiviral treatment for more than 1 year was $37.0 \%$, whereas it was $45.2 \%$ in patients who did not receive the antiviral treatment for 1 year $(P=0.023)$ (Additional file 1: Figure S1). Patients who received longer period of antiviral therapy had a lower incidence rate of rebleeding.

We also analyzed the cumulative survival rate in different time points. The survival rate at $1,2,3$, and 5 years in the antiviral group was higher than that in the non-antiviral group $(P<0.01)$ (Fig. $3 \mathrm{a}, \mathrm{b}, \mathrm{c}, \mathrm{d})$. The cumulative survival rates were $96.5,89.1,80.6$, and $59.6 \%$ in the antiviral group at $1,2,3$, and 5 years, respectively, whereas the corresponding survival rates in the nonantiviral group were 85.6, 73.5, 64.6, and $47.2 \%$, respectively. After matching, significant differences were still observed $(P<0.01)$ (Fig. 3e, f, g, h).

We also performed IPTW analysis to determine the benefits of antiviral treatment on rebleeding and survival rates. The result was that after the IPTW analysis, the rebleeding rate was lower and survival rate was higher in the antiviral group than those in the non-antiviral group $(P<0.001)$ (Fig. 4).

\section{Discussion}

To some extent of our knowledge, this study was the largest sample analysis to elucidate the role of antiviral therapy to prevent variceal rebleeding in patients with $\mathrm{CHB}$ after an endoscopic therapy.

It was widely investigated that antiviral therapy could prevent or reverse the progression of fibrosis in patients with $\mathrm{CHB}[25,26]$. Observing the 440 patients with HBVrelated cirrhosis, Goyel et al. [27] found that antiviral therapy significantly improved the Child score and patient's overall clinical course. Long-term antiviral therapy could also reduce the incidence of HCC among patients with HBV-related cirrhosis [28, 29]. A few reports focused on the effects of antiviral therapy on variceal bleeding and its outcome [30, 31]; However, no research publication elucidated the effects of antiviral therapy on patients with HBV-related cirrhosis receiving endoscopic therapy after variceal bleeding. Recently, 107 patients with $\mathrm{HBeAg}$ negative compensated cirrhosis was followed up for 12 years by Lampertico and his colleagues [32]. They found that long-term antiviral therapy in HBeAg-seronegative patients with compensated cirrhosis may significantly improve preexisting esophageal varices [32]. Consistent with the above reports, our results showed that longterm antiviral therapy significantly decreased the rebleeding rate in patients with HBV-related cirrhosis after an endoscopic therapy.

The present data showed that among the factors involved in rebleeding after an endoscopic therapy, antiviral treatment was the only protective element related with decreased rebleeding rate. The results suggested that antiviral therapy should be administrated to patients with HBV-related cirrhosis with variceal bleeding, albeit receiving an endoscopic therapy. However, as a retrospective study, some natural limitations were difficult to overcome. Firstly, not all patients were periodically followed up at 3 or 6 months after the endotherapy because of economic reasons, leading to a fact that some influential factors were not analyzed in our present study. Those factors included (but not limited to) the incidence of complications and some druginduced bias (proton-pump inhibitor, beta-receptor blocking agents, and its course of treatment). Secondly,
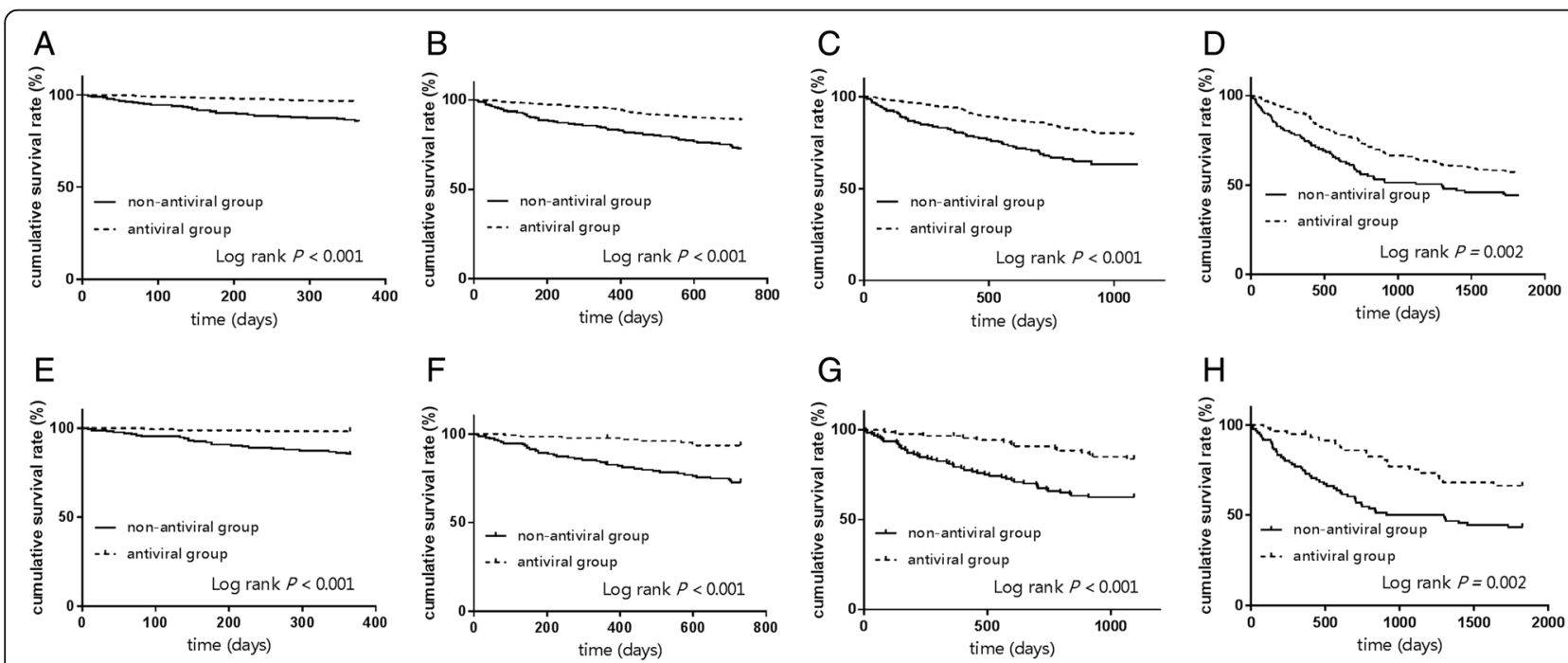

Fig. 3 The cumulative survival rates at different time points in the entire cohort ( $N=1139 ; 216$ in the non-antiviral group vs. 923 in the antiviral group) and propensity score matched cohort ( $N=348 ; 174$ in the non-antiviral group vs. 174 in the antiviral group). The entire cohort: $\mathbf{a}, 1$ year, $\mathbf{b}$, 2 year, $\mathbf{c}, 3$ year, $\mathbf{d}$, 5 year. The PS matching cohort: $\mathbf{e}, 1$ year, $\mathbf{f}, 2$ year, $\mathbf{g}, 3$ year, $\mathbf{h}$, 5 year 

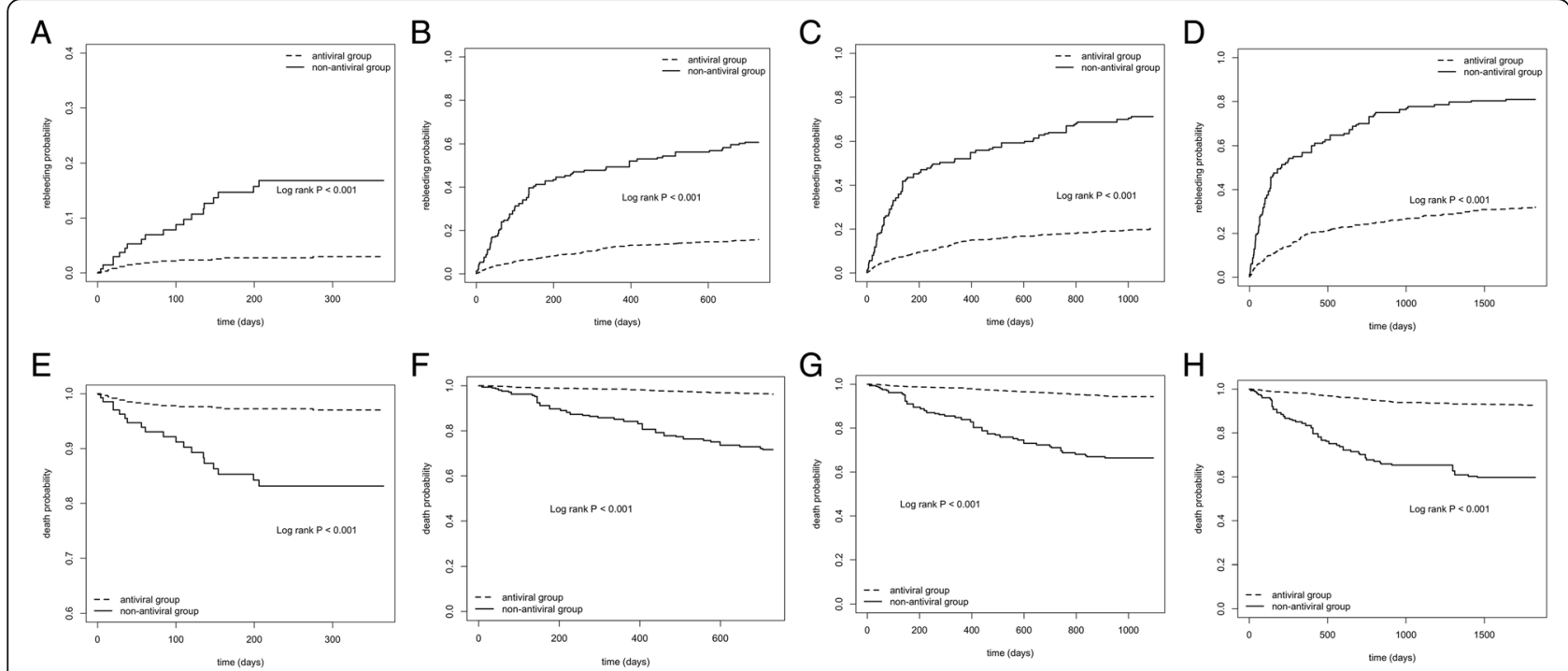

Fig. 4 The cumulative rebleeding and survival rates at different time points by IPTW analysis $(N=1139 ; 216$ in the non-antiviral group vs. 923 in the antiviral group). a, rebleeding rate at 1 year; $\mathbf{b}$, rebleeding rate at 2 years; $\mathbf{c}$, rebleeding rate at 3 years; $\mathbf{d}$, rebleeding rate at 5 years; $\mathbf{e}$, survival rate at 1 year; $\mathbf{f}$, survival rate at 2 years; $\mathbf{g}$, survival rate at 3 years; $\mathbf{h}$, survival rate at 5 years

since the varices were dynamic and might change with the endoscopic treatment (from GOV1 to GOV2), some patients might receive different endotherapy at different follow-up time, EVL, EVS, or combined therapy. Consequently, we did not analyze the relationship between the endoscopic therapies and rebleeding rate. Thirdly, as it is a retrospective study, a number of complications of HBV related cirrhosis have not been collected, such as the incidence of hepatic encephalopathy, ascites, and portal vein thrombosis. We should observe the complications of chronic HBV related cirrhosis in the prospective study in the future. Lastly, the data collected to analyze the stage of liver cirrhosis were inadequate. The liver stiffness measurement was introduced in November 2015 in our hospital, and only few patients received liver biopsy. In addition, no data were available regarding the grade of fibrosis after the therapy to analyze the study population.

Since lamivudine [33, 34], adefovir [35], entecavir [36], telbivudine [37], and tenofovir [38] all may delay or reverse liver fibrosis, we did not evaluate the differences in their efficacy. In fact, inhibiting the HBV replication is a critical factor to reverse liver fibrosis [39] and leads to decrease of the rebleeding rate in patients with HBVrelated cirrhosis with endoscopic treatment.

\section{Conclusion}

In conclusion, our present data suggested that antiviral therapy significantly reduced the rebleeding rate in patients with HBV-related cirrhosis who received endoscopic treatment after the first variceal bleeding.

\section{Additional file}

Additional file 1: Figure S1. The cumulative incidence rate of rebleeding at 1 year. $\mathrm{A}$, antiviral group $(N=923)$ and non-antiviral group $(N=216) ; B$, antiviral treatment for $>1$ year $(N=529)$ and $<1$ year $(N=$ 394). (TIF $126 \mathrm{~kb})$

\section{Abbreviations}

AFP: Alpha fetoprotein; ALB: Albumin; ALP: Alkaline phosphatase; ALT: Alanine transaminase; CHB: Chronic hepatitis B; CHE: Cholinesterase; Cr: Creatinine; EVL: Endoscopic varices ligation; EVS: Endoscopic varices sclerotherapy; GGT: Gamma-glutamyl transpeptidase; GOV: Gastroesophageal varices; HBV: Hepatitis B virus; HGB: Hemoglobin; IGV: Isolated gastric varices; IPTW: Inverse probability of treatment weighting; MELD: Model for end-stage liver disease; NSBB: Non-selective betablockers; PLT: Platelet; PT: Prothrombin time; TBIL: Total bilirubin; WBC: White blood cell

\section{Acknowledgements}

Not applicable.

\section{Authors' contributions}

$\mathrm{LH}$ and $\mathrm{XY}$ were major contributors in writing the manuscript. JM collected the data and performed statistical analysis. PL, YJ and JH collected the clinical data. JY, YZ and XL performed statistical analysis. YL collected and checked the data. HW designed the present study. All authors read and approved the final manuscript.

\section{Funding}

This study was supported by the Digestive Medical Coordinated Development Center of Beijing Hospitals Authority (XXZ0404), Capital Foundation for Clinical Characteristic Applied Research Projects (Z181100001718084) and the Study on Modernization of Traditional Chinese Medicine (2018YFC1705700) to Prof. Wei. The funding body did not play a role in the design of the study, collection, analysis, interpretation of data, and in writing the manuscript.

\section{Availability of data and materials}

The datasets used and/or analysed during the current study are available from the corresponding author on reasonable request. 


\section{Ethics approval and consent to participate}

The study was approved by the Clinical Research Ethics Committee of Beijing Ditan Hospital (No. 2018-021-01). Written consent from patients was waived by the institutional review board because of the retrospective study design.

\section{Consent for publication}

Waiver of individual patient informed consent was obtained from the institutional review board. No details, images, or videos relating to individual participants are included in the manuscript.

\section{Competing interests}

The authors declare that they have no competing interests.

\section{Author details}

'Department of gastroenterology, Beijing Ditan Hospital, Capital Medical University, No.8, Jingshun East Street, Chaoyang District, Beijing 100015, China. ${ }^{2}$ Beijing Huaxin Hospital, the First Affiliated Hospital of Tsinghua Uinversity, Beijing, China.

Received: 1 August 2018 Accepted: 11 June 2019

Published online: 21 June 2019

\section{References}

1. Loomba $R$, Liang TJ. Hepatitis B reactivation associated with immune suppressive and biological modifier therapies: current concepts, management strategies, and future directions. Gastroenterology. 2017; 152(6):1297-309.

2. Tong S, Revill P. Overview of hepatitis B viral replication and genetic variability. J Hepatol. 2016;64(1 Suppl):S4-S16.

3. Shan S, Cui F, Jia J. How to control highly endemic hepatitis B in Asia. Liver Int. 2018:38(Suppl 1):122-5.

4. Zhu RX, Seto WK, Lai CL, et al. Epidemiology of hepatocellular carcinoma in the Asia-Pacific region. Gut and liver. 2016;10(3):332-9.

5. Tanaka M, Katayama F, Kato $H$, et al. Hepatitis B and $C$ virus infection and hepatocellular carcinoma in China: a review of epidemiology and control measures. J Epidemiol. 2011;21(6):401-16.

6. Zhang QQ, An X, Liu YH, et al. Long-term nucleos(t)ide analogues therapy for adults with chronic hepatitis $B$ reduces the risk of long-term complications: a meta-analysis. Virol J. 2011;8:72.

7. Vlachogiannakos J, Papatheodoridis GV. Hepatitis B: who and when to treat? Liver Int. 2018;38(Suppl 1):71-8.

8. Marcellin P, Zoulim F, Hézode C, et al. Effectiveness and safety of Tenofovi Disoproxil fumarate in chronic hepatitis B: a 3-year, prospective, real-world study in France. Dig Dis Sci. 2016;61(10):3072-83.

9. Ghany MG. Current treatment guidelines of chronic hepatitis B: the role of nucleos(t)ide analogues and peginterferon. Best Pract Res Clin Gastroenterol. 2017;31(3):299-309.

10. Long B, Koyfman A. The emergency medicine evaluation and management of the patient with cirrhosis[J]. Am J Emerg Med. 2018;36(4):689-98. https:// doi.org/10.1016/j.ajem.2017.12.047.

11. Bosch J, Garcia-Pagan JC. Prevention of variceal rebleeding. Lancet. 2003;361:952-4.

12. Baiges A, Hernández-Gea V, Bosch J. Pharmacologic prevention of variceal bleeding and rebleeding. Hepatol Int. 2018;12(Suppl 1):68-80.

13. Garbuzenko DV. Current approaches to the management of patients with liver cirrhosis who have acute esophageal variceal bleeding. Curr Med Res Opin. 2016;32(3):467-75

14. Carbonell N, Pauwels A, Serfaty L, et al. Improved survival after variceal bleeding in patients with cirrhosis over the past two decades. Hepatology. 2004;40:652-65.

15. Reiberger T, Mandorfer M. Beta adrenergic blockade and decompensated cirrhosis. J Hepatol. 2017:66(4):849-59.

16. Mandorfer M, Reiberger T. Beta blockers and cirrhosis, 2016. Dig Liver Dis. 2017:49(1):3-10

17. Lok AS, McMahon BJ, Brown RS Jr, et al. Antiviral therapy for chronic hepatitis B viral infection in adults: a systematic review and meta-analysis. Hepatology. 2016;63(1):284-306.

18. Sundaram V, Kowdley K. Management of chronic hepatitis B infection. BMJ. 2015;351:h4263.
19. Sarin SK, Kumar M, Lau GK, et al. Asian-Pacific clinical practice guidelines on the management of hepatitis B: a 2015 update [J]. Hepatol Int. 2016;10(1):1-98.

20. Tsochatzis EA, Bosch J, Burroughs AK. Liver cirrhosis [J]. Lancet. 2014; 383(9930):1749-61.

21. Avgerinos A, Armonis A, Stefanidis G, et al. Sustained rise of portal pressure after sclerotherapy, but not band ligation, in acute variceal bleeding in cirrhosis. Hepatology. 2004;39(6):1623-30.

22. Sarin SK, Lahoti D. Management of gastric varices. Baillieres Clin Gastroenterol. 1992;6(3):527-48.

23. Hou MC, Chen WC, Lin HC, et al. A new "sandwich" method of combined endoscopic variceal ligation and sclerotherapy versus ligation alone in the treatment of esophageal variceal bleeding: a randomized trial. Gastrointest Endosc. 2001;53(6):572-8

24. R Core Team. R: a language and environment for statistical computing. Vienna: R Foundation for Statistical Computing; 2018. URL https://www.Rproject.org/

25. Rockey DC. Liver fibrosis reversion after suppression of hepatitis B virus. Clin Liver Dis. 2016;20(4):667-79.

26. Ding $Y$, Duan $S$, Ye $R$, et al. More improvement than progression of liver fibrosis following antiretroviral therapy in a longitudinal cohort of HIVinfected patients with or without HBV and HCV co-infections. J Viral Hepat. 2017;24(5):412-20

27. Goyal SK, Dixit VK, Shukla SK, et al. Prolonged use of tenofovir and entecavir in hepatitis B virus-related cirrhosis. Indian J Gastroenterol. 2015;34(4):286-91.

28. Kim WR, Loomba R, Berg T, et al. Impact of long-term tenofovir disoproxil fumarate on incidence of hepatocellular carcinoma in patients with chronic hepatitis B. Cancer. 2015;121(20):3631-8.

29. Boettler T, Thimme R. Antiviral therapy in hepatitis B virus-associated liver cirrhosis. Dig Dis. 2015;33(4):608-12.

30. Li CZ, Cheng LF, Li QS, et al. Antiviral therapy delays esophageal variceal bleeding in hepatitis B virus-related cirrhosis. World J Gastroenterol. 2013; 19(40):6849-56.

31. Jwa HY, Cho YK, Choi EK, et al. Regression of esophageal varices during entecavir treatment in patients with hepatitis-B-virus-related liver cirrhosis. Clin Mol Hepatol. 2016:22(1):183-7.

32. Lampertico $P$, Invernizzi $F$, Viganò $M$, et al. The long-term benefits of nucleos(t)ide analogs in compensated HBV cirrhotic patients with no or small esophageal varices: a 12-year prospective cohort study. J Hepatol. 2015;63(5):1118-25.

33. Idilman R, Cinar K, Seven $G$, et al. Hepatitis B surface antigen seroconversion is associated with favourable long-term clinical outcomes during lamivudine treatment in $\mathrm{HBeAg-negative} \mathrm{chronic} \mathrm{hepatitis} \mathrm{B} \mathrm{patients.} \mathrm{J} \mathrm{Viral}$ Hepat. 2012;19(3):220-6.

34. $\mathrm{Xu} \mathrm{B}$, Lin $\mathrm{L}$, Xu G, et al. Long-term lamivudine treatment achieves regression of advanced liver fibrosis/cirrhosis in patients with chronic hepatitis B. J Gastroenterol Hepatol. 2015;30(2):372-8

35. Marcellin P, Chang TT, Lim SG, et al. Long-term efficacy and safety of adefovir dipivoxil for the treatment of hepatitis $B$ e antigen-positive chronic hepatitis B. Hepatology. 2008;48(3):750-8.

36. Wu IC, Lai $\mathrm{CL}$, Han $\mathrm{SH}$, et al. Efficacy of entecavir in chronic hepatitis $\mathrm{B}$ patients with mildly elevated alanine aminotransferase and biopsy-proven histological damage. Hepatology. 2010;51(4):1185-9.

37. Hou JL, Xu D, Shi G, et al. Long-term Telbivudine treatment results in resolution of liver inflammation and fibrosis in patients with chronic hepatitis B. Adv Ther. 2015:32(8):727-41.

38. Buti M, Tsai N, Petersen J, et al. Seven-year efficacy and safety of treatment with tenofovir disoproxil fumarate for chronic hepatitis B virus infection. Dig Dis Sci. 2015;60(5):1457-64

39. Testoni B, Durantel D, Zoulim F. Novel targets for hepatitis B virus therapy. Liver Int. 2017;37(Suppl 1):33-9.

\section{Publisher's Note}

Springer Nature remains neutral with regard to jurisdictional claims in published maps and institutional affiliations. 